\title{
Improving Sound Absorption Through Nonlinear Active Electroacoustic Resonators
}

\author{
Xinxin Guo $\odot,{ }^{1,}{ }^{*}$ Hervé Lissek $\odot,{ }^{1, \dagger}$ and Romain Fleury ${ }^{2, \ddagger}$ \\ ${ }^{1}$ Laboratoire de Traitement des Signaux LTS2, Ecole Polytechnique Fédérale de Lausanne, 1015 Lausanne, \\ Switzerland
${ }^{2}$ Laboratory of Wave Engineering, Ecole Polytechnique Fédérale de Lausanne, 1015 Lausanne, Switzerland
}

(Received 17 July 2019; revised manuscript received 19 September 2019; published 10 January 2020)

The absorption of airborne noise at frequencies below $300 \mathrm{~Hz}$ is a particularly vexing problem due to the absence of natural sound-absorbing materials at these frequencies. The prevailing solution for low-frequency sound absorption is the use of passive narrow-band resonators, the absorption level and bandwidth of which can be further enhanced using nonlinear effects. However, these effects are typically triggered at high intensity levels, without much control over the form of the nonlinear absorption mechanism. In this study, we propose, implement, and experimentally demonstrate a nonlinear active control framework on an electroacoustic resonator prototype, allowing for unprecedented control over the form of nonlinearity and arbitrarily low intensity thresholds. More specifically, the proposed architecture combines linear feedforward control on the front pressure through a first microphone located at the front face of the loudspeaker and nonlinear feedback control on the membrane displacement estimated through the measurement of the pressure inside the back cavity with a second microphone located in the enclosure. It is experimentally shown that even at a weak excitation level, it is possible to observe and control the nonlinear behavior of the system. Taking the cubic nonlinearity as an example, we demonstrate numerically and experimentally that in the low-frequency range $(50-500 \mathrm{~Hz})$, the nonlinear control law allows improvement of the absorption performance, i.e., enlarging the bandwidth of optimal sound absorption while increasing the maximal absorption coefficient value and producing only a negligible amount of nonlinear distortion. The reported experimental methodology can be extended to implement various types of hybrid linear and/or nonlinear controls, thus opening new avenues for managing wave nonlinearity and achieving nontrivial wave phenomena.

DOI: 10.1103/PhysRevApplied.13.014018

\section{INTRODUCTION}

Broadband sound absorption, especially at low frequencies, still remains a challenge in both scientific research and engineering practice. Conventional sound-absorbing materials, such as porous and fibrous media [1,2], are not efficient for achieving effective absorption at low frequencies with thin layers, due to the causal nature of the acoustic response, which dictates a sum rule relating the absorption spectrum and the sample thickness [3-7]. Moreover, for any passive, linear, and time-invariant system, the bandwidth and the absorption efficiency are mutually constrained, consistent with the "Bode-Fano criterion" [8-11]. The bypassing of such inherent bounds by revoking their underlying assumptions has allowed the design of wideband matching devices [12-14].

\footnotetext{
*xinxin.guo@epfl.ch

†herve.lissek@epfl.ch

†romain.fleury@epfl.ch
}

Amongst these assumptions, passivity was the first to be considered. It can be violated by actively controlling the acoustic features of systems $[12,13]$. Such control, applied to electroacoustic resonators (ERs) to enable impedance adjustment, allows for broadening of the sound absorption, especially in the low-frequency range [15-19]. A wide range of achievable acoustic impedances can be provided by the concept, including the synthesis of narrowband single-degree-of-freedom (SDOF) resonators [19], resonances with multiple degrees of freedom $[17,18]$, or a high degree of reconfigurability [20]. Such tunability is key in many applications, such as room mode damping [21,22] or aircraft-engine tonal-noise reduction [23]. Recently, active control has also received a surge of interest as a tool for designing acoustic metamaterials (AMMs) that overcome the restrictions imposed by passive AMMs [24-27], thereby expanding the reach of metamaterial science to a wealth of nontrivial acoustic phenomena such as $\mathcal{P} \mathcal{T}$-symmetry scattering [28-31], wavefront shaping [32-34], and non-Hermitian wave control [35,36]. 
A notable technique for active impedance control uses a conventional loudspeaker, the acoustic impedance of which can be modified either by shunting its electric terminals with an engineered electric load $[15,19,22]$ or by feeding back a current or voltage proportional to a combination of the sensed acoustic quantities [16,22,37].

In the field of active electroacoustic resonators (AERs), most of the previous studies have been carried out under the assumption that the acoustic parameter fluctuations involved have been small enough to ensure that they remained linear at low frequencies. Nevertheless, nonlinear resonators also exhibit interesting performance that contributes to a variety of wave phenomena. For instance, a primary linear resonator coupled with a purely nonlinear resonator, known as a nonlinear energy sink (NES) [38-41], enables vibration extinction of the linear system, a phenomenon called energy pumping or targeted energy transfer [42-46]. Typical nonlinear effects such as higher-harmonic generation have been demonstrated and investigated in metamaterials made of nonlinear resonators [47-49]. However, the aforementioned systems usually do not allow tunable nonlinear behavior, especially at low intensities, and are typically associated with large intensity thresholds. Unlike the case of electromagnetic signals, for which nonlinearity has been exploited and incorporated with active control [50,51], the possibility of creating acoustic resonators with a tunable nonlinear response [51,52] has been left largely unexplored, except in a recent numerical study by Bitar et al. [53].

In this paper, we establish both numerically and experimentally a nonlinear control methodology that enables us to achieve a controllable nonlinear SDOF AER, which exhibits nonlinear effects even at weak excitation levels. This is obtained through a current-driven feedbackcontrol framework applied on a closed-box electrodynamic loudspeaker. Focusing on the absorption performance, we use our findings and determine proper control laws that allow the improvement of sound absorption, while producing only a negligible amount of nonlinear distortion.

The paper is organized as follows. Based on the known linear theory of active impedance control on the ER, a nonlinear control strategy is first introduced in Sec. II, together with the definition of a relevant absorption performance metric. The prototype and the corresponding experimental setup are then described in Sec. III. Thereafter, the absorption performance of the achieved nonlinear AER prototype is examined. Two different types of control law are considered; a purely nonlinear control law (Sec. IV) and hybrid control laws that combine different linear settings with the proposed nonlinear one (Sec. V). Simulation through a time-domain integration method is also implemented in order to verify and validate the experimental results.

\section{NONLINEAR SINGLE DEGREE-OF-FREEDOM ELECTROACOUSTIC RESONATOR}

\section{A. Description and working principle}

In the low-frequency range and under weak excitation, an electrodynamic loudspeaker behaves as a linear SDOF ER. The mechanical part of the loudspeaker can simply be modeled as a conventional mass-spring-damper system, where the moving diaphragm of mass $M_{\mathrm{ms}}$ is attached through an elastic suspension of mechanical compliance $C_{\mathrm{ms}}$ and the global losses are accounted for in the mechanical resistance $R_{\mathrm{ms}}$. In the present work, we consider a loudspeaker mounted in an enclosure of volume $V_{b}$. Figure 1 illustrates the schematic representation and the circuit analogy of the closed-box loudspeaker. Denoting the effective area of the loudspeaker diaphragm by $S_{d}$ and the force factor of the moving-coil transducer by $B l$, Newton's second law, applied to the loudspeaker diaphragm, reads

$$
\begin{aligned}
M_{\mathrm{ms}} \frac{d v(t)}{d t}= & S_{d}\left[p_{f}(t)-p_{b}(t)\right]-R_{\mathrm{ms}} v(t)-\frac{1}{C_{\mathrm{ms}}} \int v(t) d t \\
& -B \operatorname{li}(t),
\end{aligned}
$$

where $p_{f}(t)$ and $p_{b}(t)$ designate the acoustic pressures applied, respectively, to the front and the rear faces of the membrane, whereas $v(t)$ and $i(t)$ represent the acoustic velocity of the diaphragm and the current circulating in the moving coil, respectively.

At low frequencies, the sound pressure inside the cavity of volume $V_{b}$ is assumed uniform, yielding a linear relation between the rear pressure $p_{b}(t)$ and the displacement of the diaphragm $\xi(t)=\int v(t) d t$, i.e.,

$$
p_{b}(t) \cong \frac{S_{d}}{C_{\mathrm{ab}}} \xi(t),
$$

where $C_{\mathrm{ab}}=V_{b} /\left(\rho c^{2}\right)$ represents the acoustic compliance of the enclosure, with $\rho$ and $c$ denoting the air-mass density and the associated speed of sound. Introducing the overall mechanical compliance $C_{\mathrm{mc}}=C_{\mathrm{ms}} C_{\mathrm{ab}} /\left(S_{d}^{2} C_{\mathrm{ms}}+C_{\mathrm{ab}}\right)$ accounting for the fluid compressibility on the rear face of

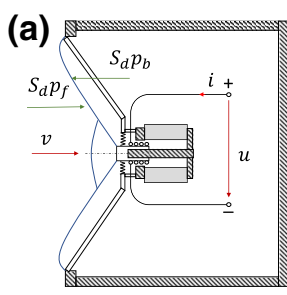

(b)

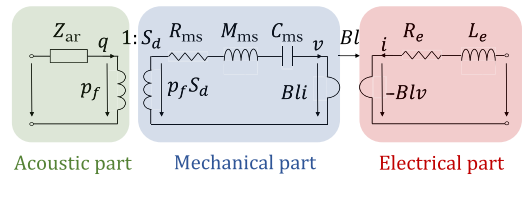

FIG. 1. A schematic representation (a) and a circuit analogy (b) of the considered closed-box electrodynamic loudspeaker system [22]. 
diaphragm, Eq. (1) can be rewritten as

$$
M_{\mathrm{ms}} \frac{d^{2} \xi(t)}{d t^{2}}=p_{f}(t) S_{d}-R_{\mathrm{ms}} \frac{d \xi(t)}{d t}-\frac{1}{C_{\mathrm{mc}}} \xi(t)-B \operatorname{Bl}(t) .
$$

In the open-circuit configuration, i.e., $i=0$, the frequency response of the considered ER is characterized by the specific acoustic impedance $Z_{\mathrm{as}}$ defined in the linear regime by

$$
Z_{\mathrm{as}}(j \omega)=\frac{P_{f}(j \omega)}{V(j \omega)}=j \omega M_{\mathrm{as}}+R_{\mathrm{as}}+\frac{1}{j \omega C_{\mathrm{ac}}},
$$

where $M_{\mathrm{as}}=M_{\mathrm{ms}} / S_{d}, R_{\mathrm{as}}=R_{\mathrm{ms}} / S_{d}$, and $C_{\mathrm{ac}}=C_{\mathrm{mc}} S_{d}$ are the equivalent acoustic parameters. The uppercase symbols $P_{f}$ and $V$ designate the frequency responses of the considered acoustic quantities (front pressure and velocity) to distinguish them from their denotations in the time domain (represented by lowercase symbols).

When the loudspeaker is driven with a given electrical current, the added term due to $i(t) \neq 0$ leads to an impedance response that is different from $Z_{\text {as }}$. Active impedance control is typically based on controlling the current that circulates through the loudspeaker coil. This type of control has proven to be more stable compared to others such as voltage control [22], as it offers the opportunity to tune the acoustic impedance and the absorption performance of the acoustic resonator without having to model its electrical part.

\section{B. Acoustic impedance control of a linear AER}

Before introducing the nonlinear control strategy, a case of linear control is first presented. To this end, one defines a target specific acoustic impedance $Z_{\mathrm{st}}$ that the linear AER, once controlled, is expected to present. We assume here that it takes the form of a SDOF resonator, similar to the passive impedance of Eq. (4):

$$
Z_{\mathrm{st}}(j \omega)=j \omega \mu_{1} M_{\mathrm{as}}+R_{\mathrm{st}}+\frac{\mu_{2}}{j \omega C_{\mathrm{ac}}},
$$

where $\mu_{1}, \mu_{2}$, and $R_{\mathrm{st}}$ are design parameters corresponding to the desired mass, compliance, and resistance of the controlled ER, respectively. Such target impedance parameters are used to adjust the frequency response of the resonator (the resonance frequency, quality, and damping factor). Regarding the absorption performance, it is easy to show that the maximum absorption appears at the frequency [22]

$$
f_{\mathrm{st}}=f_{s} \sqrt{\frac{\mu_{2}}{\mu_{1}}},
$$

where $f_{s}=\left(2 \pi \sqrt{M_{\mathrm{as}} C_{\mathrm{ac}}}\right)^{-1}$ is the natural resonance frequency of the ER. Thus, by adjusting the ratio $\mu_{2} / \mu_{1}$, the frequency of maximum absorption can be tuned (note that it can also be left unchanged at $f_{s}$ ). Additionally, perfect absorption can be achieved as well at the prescribed frequency $f_{\text {st }}$ if the target resistance $R_{\text {st }}$ reaches the specific acoustic impedance of air, i.e., $R_{\mathrm{st}}=Z_{c}=\rho c$.

The objective of active impedance control is to identify the controller transfer function enabling the desired conversion from the input pressure $p_{f}$, that is sensed using a microphone, to the output current $i$ that is sent back to the loudspeaker, in order to achieve the target impedance $Z_{\text {st }}$ on the ER. In the considered linear regime, the transfer function $\Phi(s)$ can be derived from Eqs. (3) and (5) in the Laplace domain (with variable $s$ ) as

$$
\Phi(s)=\frac{I_{\mathrm{L}}(s)}{P_{f}(s)}=\frac{Z_{\mathrm{st}}(s) S_{d}-Z_{\mathrm{as}}(s) S_{d}}{B l Z_{\mathrm{st}}(s)},
$$

where the symbol $I_{\mathrm{L}}$ denotes the Laplace transform of the current in the linear configuration.

Through the control of the current $I_{\mathrm{L}}$ delivered to the loudspeaker terminals as a function $\Phi(s)$ of the input front pressure $P_{f}$, the impedance and the absorption properties of the resonator can therefore be tuned. This has previously been demonstrated over a quite wide frequency range, depending on the control parameters $\left(\mu_{1}, \mu_{2}, R_{\mathrm{st}}\right)$ [15].

\section{Nonlinear control of the ER}

As previously seen in Eq. (2), the rear pressure $p_{b}$ is proportional to the displacement of the loudspeaker diaphragm in the low-frequency range. This provides the opportunity to define a current $i_{\mathrm{NL}}$ as a function of a nonlinear transformation of the rear pressure. In the present work, we propose to add a nonlinear part to the control law by driving an additional current $i_{\mathrm{NL}}$, defined as a nonlinear cubic transformation of the rear pressure $p_{b}$ :

$$
i_{\mathrm{NL}}(t)=G_{\mathrm{ui}} \beta_{\mathrm{NL}}\left[G_{\mathrm{mic}} p_{b}(t)\right]^{3} \propto \xi^{3}(t),
$$

where $\beta_{\mathrm{NL}}$ denotes the tunable nonlinear parameter, while $G_{\text {mic }}$ and $G_{\text {ui }}$ are the sensitivity of the microphone and the gain that converts the voltage into current, respectively.

Then, such a current $i_{\mathrm{NL}}$ will contribute to adding a nonlinear component to the stiffness (inverse of compliance) of the resonator, which will be fully adjustable and potentially much larger than what is possible with passive mechanical elements. Indeed, for an intrinsically nonlinear mechanical system, a relatively strong excitation is always required to trigger nonlinear effects. Here, instead, by simply increasing the nonlinear parameter $\beta_{\mathrm{NL}}$, the proposed nonlinear control methodology facilitates the emergence of nonlinear phenomena without requiring large excitation levels. The implementation and further analysis of the designed nonlinear AER are presented in Secs. IV and V, by considering either pure nonlinear control defined with $i=i_{\mathrm{NL}}$ or hybrid control with $i=i_{\mathrm{L}}+i_{\mathrm{NL}}$. 


\section{Absorption coefficient metric for linear and nonlinear AERs}

We are interested in the absorption performance of the controlled AERs. The chosen metric of interest is the absorption coefficient, which can be determined by sensing both the front pressure and the membrane axial velocity.

In the linear regime, such two quantities, measured in the time domain and processed in the frequency domain, allow the effective specific acoustic impedance of the diaphragm $Z(j \omega)=P_{f}(j \omega) / V(j \omega)$ to be easily extracted under a sweep sine excitation over the frequency range of interest. Then, the absorption coefficient $\alpha_{\mathrm{L}}(j \omega)$ can be obtained in a straightforward manner through the conventional relationship:

$$
\alpha_{\mathrm{L}}(j \omega)=1-\left|\frac{Z(j \omega)-Z_{c}}{Z(j \omega)+Z_{c}}\right|^{2}
$$

where $Z_{c}=\rho c$ denotes the specific acoustic impedance of air.

However, for a nonlinear system, the energy transfer from fundamental frequency $\omega$ to higher harmonics $(n \omega$ with $n \geq 2$ ) should be additionally taken into account, leading to a generalized definition of the absorption coefficient as

$$
\alpha_{\mathrm{NL}}=1-\sum_{n=1}^{n=N}\left|R_{n}\right|^{2}=\alpha_{\mathrm{L}}-\sum_{n=2}^{n=N}\left|R_{n}\right|^{2},
$$

where $R_{n}$ represents the complex pressure amplitude of the generated $n$th harmonic normalized by that of the fundamental incoming wave.

\section{EXPERIMENTAL SETUP}

In our experiment, a commercially available electrodynamic loudspeaker (Monacor SPX-30M), mounted with an enclosure having a lateral surface of $12 \times 12 \mathrm{~cm}^{2}$ and with a thickness of $6.8 \mathrm{~cm}$, is employed as the experimental ER prototype. The overall closed-box ER presents a resonance frequency around $200 \mathrm{~Hz}$, corresponding to a wavelength of $1.7 \mathrm{~m}$, which is 25 times larger than the cavity dimensions, confirming the subwavelength nature of the absorber. Moreover, note that the definition of the linear control law requires knowledge of the mechanical parameters $M_{\mathrm{ms}}, R_{\mathrm{ms}}$ and $C_{\mathrm{mc}}$ as well as the force factor $B l$ of the considered ER [see Eqs. (5) and (7)]. These parameters are determined from two calibration measurements of the acoustic impedance, the first obtained with the ER in open circuit and the second in the short-circuit case, as presented in Ref. [22]. The extracted loudspeaker parameters, as well as the estimated effective area $S_{d}$ of the loudspeaker diaphragm, are summarized in Table I.
TABLE I. The estimated Thiele-Small parameters of the closed-box Monacor SPX-30M lousdpeaker.

\begin{tabular}{lccccc}
\hline \hline Parameter & $M_{\mathrm{ms}}$ & $R_{\mathrm{ms}}$ & $C_{\mathrm{mc}}$ & $B \ell$ & $S_{d}$ \\
Unit & $\mathrm{g}$ & $\mathrm{N} \mathrm{s} \mathrm{m}^{-1}$ & $\mathrm{~mm} \mathrm{~N}^{-1}$ & $\mathrm{~N} \mathrm{~A}^{-1}$ & $\mathrm{~cm}^{2}$ \\
Value & 2.7 & 0.4516 & 0.2185 & 3.3877 & 32 \\
\hline \hline
\end{tabular}

For implementing the desired controls, two PCB Piezotronics Type 130D20 ICP microphones (nominal sensitivity $G_{\text {mic }}=45 \mathrm{mV} \mathrm{Pa}^{-1}$ ) are employed for sensing, respectively, the front pressure $p_{f}$ and the rear pressure $p_{b}$ of the loudspeaker diaphragm, as illustrated in Fig. 2. In the case of purely nonlinear (or linear) control, only the measured rear (or front) pressure is used by the control system to generate an output current to the ER, whereas to achieve hybrid active control, both pressures $p_{f}$ and $p_{b}$ are used. The control law is operated through a National Instruments CompactRio field-programmable gate-array (FPGA) platform, set via LABVIEW 2017 (32-bit). The current-drive amplifier feeding back the ER enables the conversion from voltage to current, with a gain of $G_{\mathrm{ui}} \approx$ $9.63 \mathrm{~mA} \mathrm{~V}^{-1}$.

For the acoustic measurements, a Tannoy loudspeaker driven by a signal generator is employed for exciting the whole system. The front pressure is sensed with the same microphone that is used for the control implementation (placed near the front face of the loudspeaker). The membrane axial velocity of the ER is captured by a laser vibrometer focused on the loudspeaker diaphragm. Depending on the definition of the absorption coefficient (accounting for the nonlinear effects or not), the absorption performance of the developed AER can be properly characterized through a judicious measurement of the velocity of the diaphragm and the pressure in front of it.

Since the assessment of the developed nonlinear AERs needs a comparison with the linear ones, we use both definitions of the absorption coefficient given in Sec. II D. For the linear cases, the absorption coefficient $\alpha_{\mathrm{L}}$ is determined

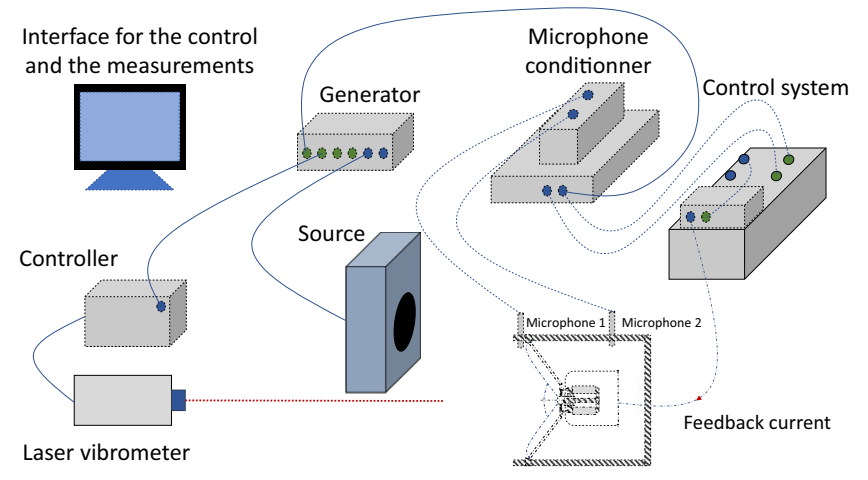

FIG. 2. The experimental setup used for applying the feedback current control on the considered closed-box loudspeaker. 
from the frequency-domain measurements. While a bidirectional sweep sine from $20 \mathrm{~Hz}$ to $820 \mathrm{~Hz}$ with sweep rate of 0.02 decades per second is delivered to the sound source, the transfer function between the front sound pressure $p_{f}$ and the membrane velocity $v$ is first estimated. $\alpha_{\mathrm{L}}$ is then derived according to Eq. (9). In the nonlinear cases, the absorption coefficient $\alpha_{\mathrm{NL}}$ is determined from time-domain measurements, since it is necessary to extract the pressure amplitudes of all generated harmonics. To this end, a stepwise monochromatic sine excitation with varying frequency within the range of interest $(50-500 \mathrm{~Hz})$ is used. For the sake of simplicity, a fine frequency step of $2 \mathrm{~Hz}$ is employed around the resonance of the ER and a coarser frequency step of $10 \mathrm{~Hz}$ is employed further away from the resonance. The time signals are acquired and recorded with resolution of $78 \mu \mathrm{s}$ and a duration of $10 \mathrm{~s}$. Based on these measurements, the Fourier transform allows amplitude estimation of all harmonic components of the measured quantities. Thus the absorption coefficient $\alpha_{\mathrm{NL}}$ can be derived according to Eq. (10). However, the incident pressure amplitude needs to be estimated in advance over the whole frequency of interest, which we proceed to do in the calibration step described in the following.

In various studies of nonlinear resonant systems reported so far, strong input intensities are typically required to trigger nonlinearities, such as the NES, where the sound pressure levels for activation and observation of nonlinear effects are in the range of $160 \mathrm{~dB}(1 \mathrm{kPa})[43,46]$. Conversely, in the presented work, we focus on excitation levels that are 3 orders of magnitude weaker, corresponding to a maximum pressure amplitude in the range of $1 \mathrm{~Pa}$ in front of the AER. Consequently, the generated nonlinear effect only results from the proposed active control. Then, in order to calibrate the incident sound pressure delivered to the ER, the sound pressure is measured near the diaphragm with the ER set to be perfectly absorbent, at different frequencies within the range of interest. In that view, several control laws are applied to the ER, so that it behaves as a narrow-band perfect absorber with various central frequencies, i.e., with $R_{\mathrm{st}}=Z_{c}$ and with different values of $\left(\mu_{1}, \mu_{2}\right)<1$. These settings allow us to achieve $\alpha_{\mathrm{L}}>0.99$ over each segmented frequency range, respectively. In this way, an anechoic termination can be provided in an active manner for the whole frequency range of interest $(50-500 \mathrm{~Hz})$. Figure 3 presents the incident sound pressure levels $(\mathrm{dB})$ measured in front of the diaphragm, at all considered frequencies when the ER is set to be absorbent, with the sound source located at a fixed distance from the ER. It can be seen from Fig. 3 that the incident pressure presents an amplitude around $1.1 \mathrm{~Pa}(94.8 \mathrm{~dB})$, especially in the range of $100-400 \mathrm{~Hz}$, where the nonlinear effect is strong. These obtained incident pressure levels are exploited in the following as a reference to derive the proportion of energy reflected through higher harmonics ( $n \omega$ with $n \geq 2$ ).

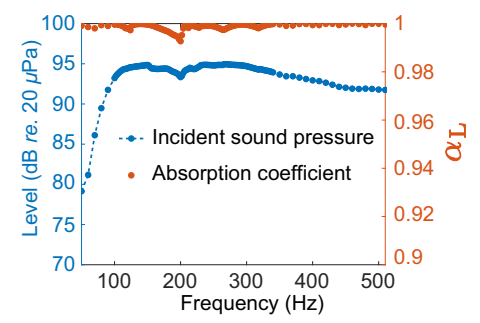

FIG. 3. The incident sound pressure level $(\mathrm{dB})$ measured with the microphone close to the loudspeaker diaphragm when nearly perfect absorption $\left(\alpha_{\mathrm{L}}>0.99\right)$ is achieved with linear AERs over the frequency range of interest $(50-500 \mathrm{~Hz})$.

\section{NONLINEAR IMPEDANCE CONTROL}

A first preliminary test is performed to validate the proportionality between the measured rear pressure $p_{b}$ and the diaphragm displacement $\xi$, as assumed in Eq. (2). For that, the transfer function defined in the frequency domain as $H_{p \xi}=P_{b}(j \omega) / \Xi(j \omega)=j \omega P_{b} / V$, where $\Xi(j \omega)$ denotes the frequency response of the displacement $\xi(t)$, is estimated for frequencies under $500 \mathrm{~Hz}$ and is shown in Fig. 4. The measurement confirms that this transfer function is almost constant in the frequency range of interest and that the proportionality factor can be averaged to $925 \times 10^{3} \mathrm{~Pa} \mathrm{~m}^{-1}$.

After validation of the required linear relation, a pure nonlinear control law defined with $i=i_{\mathrm{NL}}$ [Eq. (8)] is applied to the ER. Figure 5 shows the different experimental results achieved when the control is off $\left(\beta_{\mathrm{NL}}=0\right)$ and when it is on $\left(\beta_{\mathrm{NL}}=20\right)$, respectively. For a better comparison between the two cases, the whole measurements are carried out in the time domain under stepwise sine excitations. Since the sound source is located at a fixed position, the same incident excitation amplitude as the one measured during the calibration phase is considered, namely as low as $1.1 \mathrm{~Pa}$ in front of the ER, as shown in Fig. 3. Figures 5(a) and 5(c) present the corresponding linear frequency responses. The amplitudes of the second and third harmonics are also extracted for both control

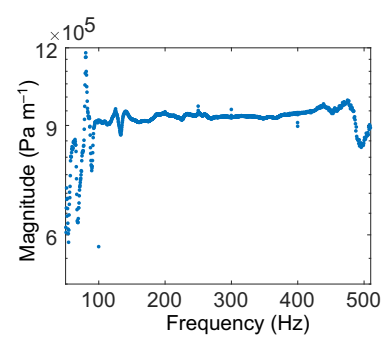

FIG. 4. The magnitude of the measured transfer function $H_{p \xi}$ between the rear pressure $p_{b}(\mathrm{~Pa})$ and the loudspeaker diaphragm displacement $\xi(\mathrm{m})$ within the frequency range $50-500 \mathrm{~Hz}$. 
(a)

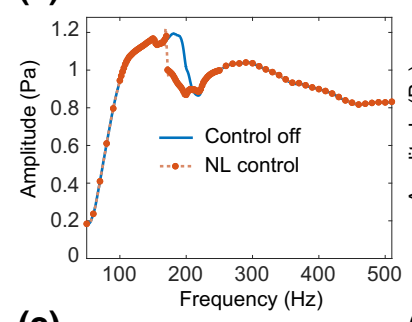

(c)

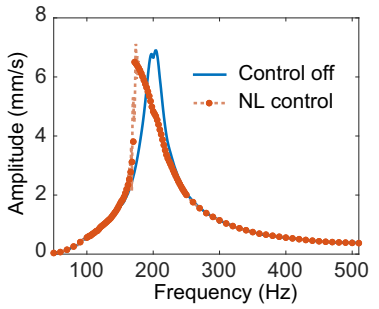

(b)

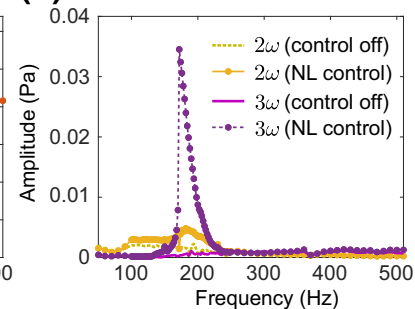

(d)

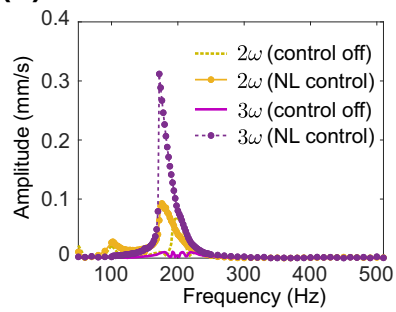

(a)

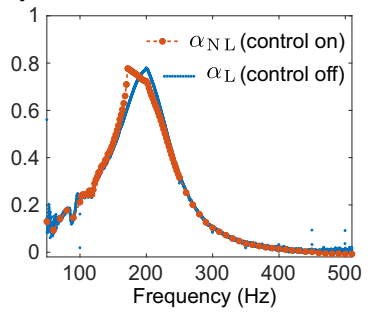

(b)

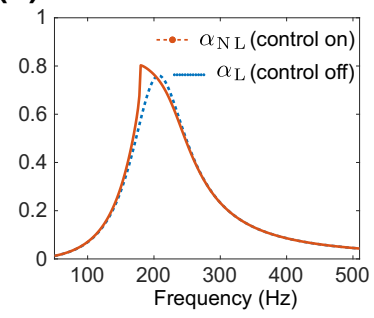

FIG. 6. The absorption curves of the achieved nonlinear AER, obtained with the definition of the absorption coefficient given in Eq. (10), adapted to nonlinear systems. The same configurations are considered as in Fig. 5, with the nonlinear parameter set as $\beta_{\mathrm{NL}}=0$ (blue dotted line) and $\beta_{\mathrm{NL}}=20$ (red dash-dotted lines), respectively. Both the experimental (a) and the simulation (b) results are presented.

FIG. 5. The nonlinear frequency responses of the measured acoustic pressure in front of the AER and of the sensed diaphragm velocity, under control off $\left(\beta_{\mathrm{NL}}=0\right)$ and under pure nonlinear control on $\left(\beta_{\mathrm{NL}}=20\right)$, respectively. The fundamental wave components of the front pressure and the diaphragm velocity are extracted from time-domain measurements at each excitation frequency $\omega$ and are shown in (a) and (c), respectively, while the amplitudes of the second and the third harmonic (at frequency $2 \omega$ and $3 \omega$, respectively) are also extracted and are presented in (b) for pressure and in (d) for velocity.

cases and are reported in Figs. 5(b) and 5(d) for the front total pressure and the diaphragm velocity, respectively.

When the control is off, the (linear) resonance of the SDOF ER can be clearly identified at the expected frequency $(200 \mathrm{~Hz})$ in the linear frequency response of the diaphragm velocity [see Fig. 5(c)]. Although the (ER) loudspeaker is not perfectly linear, the generated higher harmonics remain negligible in the passive case, with a maximum pressure amplitude of less than two thousandths of the fundamental wave, as can be seen in Fig. 5(b). The chosen weak incident pressure level (approximately 1.1 Pa) ensures that the amplitude of the total pressure also remains in the range of $1 \mathrm{~Pa}$ (with a maximum value of $1.2 \mathrm{~Pa}$ ), even around the resonance. When the nonlinear active control is on, a typical nonlinear resonance frequency shift can be observed [see Fig. 5(c)]. The thirdharmonic component is significantly increased around the nonlinear resonance of the AER owing to the defined cubic nonlinear control law, enabling a normalized maximum pressure amplitude of around 0.035 with respect to the incident wave, which corresponds to an energy proportion of nearly $0.13 \%$.

Moreover, a slight increase of the second harmonic can also be observed when the active control is on, around the nonlinear resonance, and around $100 \mathrm{~Hz}$, i.e., at half of the natural resonance frequency. Indeed, an excitation at $100 \mathrm{~Hz}$ enables the frequency match between the second

harmonic and the resonance of the ER, favoring the manifestation of $2 \omega$ even without external control, as shown in Figs. 5(b) and 5(d). This phenomenon has already been revealed in previous theoretical and numerical works on various subwavelength resonators $[47,48]$. However, the third harmonic remains largely dominant around the nonlinear resonance of the AER [see Figs. 5(b) and 5(d)]. Therefore, the applied nonlinear active control actually favors the manifestation of a cubic nonlinear resonator, as intended in the specified control law. Otherwise, comparing with the illustrated second and third harmonics, other higher harmonics $(n>3)$ are even weaker, with maximum amplitudes less than a fortieth of that of the third harmonic, thus they are not reported here.

The focus is hereafter placed on the effect of the nonlinear control on the absorption properties of the achieved nonlinear AER. From the Fourier analysis of the measured pressure and velocity shown in Fig. 5, the linear part of the absorption coefficient defined in Eq. (9), denoted as $\alpha_{\mathrm{L}}$, can be determined by extracting the fundamental components [Figs. 5(a) and 5(c)]. Then, the pressure amplitudes of the second and third harmonics of Fig. 5(b), divided, respectively, by that of the incident wave presented in Fig. 3, allow the estimation of the energy proportion reflected through these higher harmonics. Thus, following Eq. (10), the desired absorption coefficient $\alpha_{\mathrm{NL}}$ can be derived by subtracting the above part of the reflected energy from the linear part of the absorption. For the same cases of control on and off as considered in Fig. 5 (identified by $\beta_{\mathrm{NL}}=20$ and $\beta_{\mathrm{NL}}=0$, respectively), Fig. 6(a) illustrates the derived absorption coefficient ( $\alpha_{\mathrm{L}}$ for the passive case, $\alpha_{\mathrm{NL}}$ for the nonlinear case) under the same weak excitation level as before.

This comparison between two such cases shows that the achieved nonlinear AER allows primarily for broadening the absorption bandwidth toward low frequency in the vicinity of the resonance, corresponding to an increase in 
the impedance bandwidth (measured as the bandwidth over which the ER impedance magnitude is lower than $\sqrt{2} Z_{\min }$, where $Z_{\min }$ is the minimum magnitude of the impedance) from around $23 \mathrm{~Hz}$ to $35 \mathrm{~Hz}$, namely an increase of $50 \%$. The optimal absorption improvement occurs at the nonlinear resonance frequency $(172 \mathrm{~Hz})$, where $\alpha_{\mathrm{NL}}$ increases from 0.61 to 0.78 . However, according to the frequency responses of all generated harmonics presented in Fig. 5, only a tiny fraction of energy is transferred into higher harmonics, with a maximum proportion of only $0.13 \%$. The nonlinear effect introduced by the proposed active control manifests mainly in enhancing sound absorption around the resonance of the AER, while producing only negligible distortion. The absorption curve with the definition of $\alpha_{\mathrm{NL}}$ including the energy radiation of all generated harmonics [e.g., Fig. 6(a)], in parallel with the frequency responses of the nonlinear components [e.g., Fig. 5(b)], allows for a complete assessment of the nonlinear effect on the absorption performance of the achieved AER. The combination of these two types of acoustic quantities will thus be considered for illustrating the results of all the following, more advanced, control configurations.

In order to validate that the observed nonlinear behavior results from the defined nonlinear control rule, a numerical simulation based on the classical fourth-order Runge-Kutta (RK4) integration method [54] is herein implemented via MATLAB. In the simulation, the time delay $\tau$ between the input and the output of the control system is accounted for, since such a delay can make the resulting absorption coefficient different from the one obtained directly via Eq. (3). Thus, with the defined feedback current $i(t)=$ $G_{\mathrm{ui}} \beta_{\mathrm{NL}}\left(p_{d}(t) G_{\mathrm{mic}}\right)^{3} \propto \xi^{3}(t)$, the full problem under consideration is described by the modified motion equation as

$$
\begin{aligned}
M_{\mathrm{ms}} \frac{d^{2} \xi(t)}{d t^{2}}= & p_{f}(t) S_{d}-R_{\mathrm{ms}} \frac{d \xi(t)}{d t}-\frac{1}{C_{\mathrm{mc}}} \xi(t) \\
& -B \operatorname{Bli}(t-\tau) H(t-\tau),
\end{aligned}
$$

where $H(t-\tau)$ is the Heaviside function, which equals 1 for $t \geq \tau$ and zero otherwise.

For the sake of accuracy, a stepwise monochromatic source with a duration of $20 \mathrm{~s}$ at each frequency step is considered in the simulations. For each discrete frequency, the absorption coefficient as defined in Eq. (9) is derived from the total front acoustic pressure and the velocity, which are determined by solving numerically the above motion equation, Eq. (10). Regarding the considered time delay, using a sweep step of $1 \times 10^{-5} \mathrm{~s}$ in the simulation, it is found to be $\tau=6 \times 10^{-5} \mathrm{~s}$ by fitting the experimental results. Figure 6(b) shows the simulation results of the defined absorption coefficient for both control off $\left(\beta_{\mathrm{NL}}=0\right)$ and pure nonlinear control cases $\left(\beta_{\mathrm{NL}}=20\right)$, under sine excitation performed with a frequency step of $2 \mathrm{~Hz}$ in the range of $50-500 \mathrm{~Hz}$.
Although the estimation method employed for extracting the physical parameters $\left(M_{\mathrm{ms}}, C_{\mathrm{mc}}, R_{\mathrm{ms}}\right.$, and $\left.B l\right)$ could be further improved, the comparison between the experiments and simulations on the absorption curve, as well as the investigation of the generation of higher harmonics, is excellent.

Finally, note that in both Figs. 5 and 6, the tested nonlinear configuration corresponds to a value $\beta_{\mathrm{NL}}=20$, identified as the threshold above which saturation occurs under the considered excitation level. We additionally verify that the same absorption curve can be obtained at lower excitation levels simply by increasing the value of $\beta_{\mathrm{NL}}$, which is a clear advantage compared to other passive nonlinear systems reported in the literature. While the bandwidth increase toward low frequency is considerable, the performed nonlinear control can be further controlled and improved by combining it with a linear one. Section $\mathrm{V}$ will show how such hybrid control allows the absorption performance of the ER to be improved compared to the case of pure nonlinear control.

\section{COMBINATION OF LINEAR AND NONLINEAR IMPEDANCE CONTROL LAWS}

Different linear active control laws are taken into account in this section and combined with the previously presented nonlinear control. This allows us to modify the whole dynamics of the ER. More specifically, the resonance frequency (at which the absorption coefficient is maximal or the impedance is minimal and purely resistive) can be tuned through the ratio of linear control parameters $\mu_{2} / \mu_{1}$. Moreover, it has been demonstrated that the absorption bandwidth depends primarily on the amount $S_{d} / M_{\text {as }}$ [22]. Accordingly, this section considers the variation of the design parameter $\mu_{2}$ assigned to the compliance, while choosing the mass factor $\mu_{1}=1$ so that the bandwidth of absorption of the ER remains nearly unchanged in the linear regime. A brief discussion about the mass factor is given at the end of this section.

First, a linear control law with $\mu_{1}=1$ and $\mu_{2}=1.5$ is considered. It maintains the original absorption bandwidth of the ER but shifts the maximum absorption slightly, from $200 \mathrm{~Hz}$ to $240 \mathrm{~Hz}$. Regarding the target resistance $R_{\mathrm{st}}$, a total absorption $(\alpha=1)$ is achievable at the target frequency $f_{\mathrm{st}}$ when $R_{\mathrm{st}}$ coincides with the specific acoustic impedance of air $Z_{c}$. With such a linear configuration, the nonlinearity provided by the hybrid control can only enable the enlargement of the absorption bandwidth. Hence, with a view to assessing the overall effect of the resulting nonlinearity, we first assign a target resistance that is different from $Z_{c}$ for the present linear control law (defined by $\mu_{1}=1$ and $\mu_{2}=1.5$ ), i.e., $R_{\mathrm{st}}=0.5 Z_{c}$.

The desired hybrid control is identified by the feedback current being $i(t)=i_{\mathrm{L}}(t)+i_{\mathrm{NL}}(t)$, with the linear part $i_{\mathrm{L}}(t)$ satisfying the aforementioned target impedance 
(a)

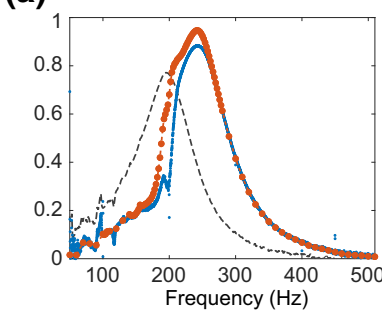

--- $\alpha_{\mathrm{L}}$ (control off) $-\alpha_{\mathrm{L}}$ (linear control)

-.-- $\alpha_{\mathrm{NL}}$ (hybrid control)

(c)

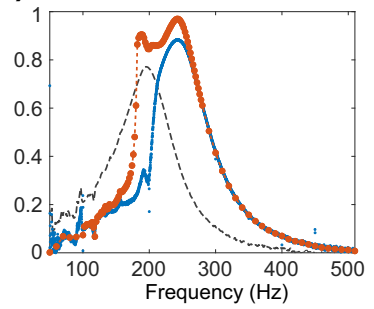

(b)

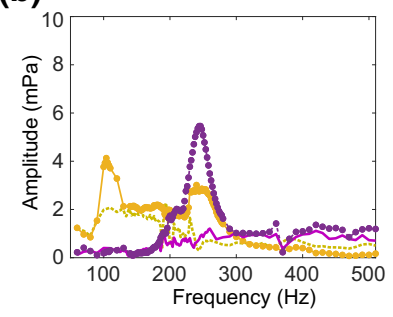

-...... $2 \omega$ (control off) $-3 \omega$ (control off)

$\rightarrow 2 \omega$ (control on) $\cdots 3 \omega$ (control on)

(d)

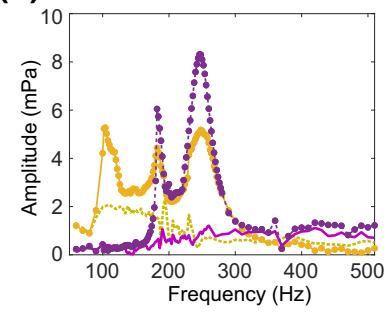

FIG. 7. The absorption curves [with $\alpha_{\mathrm{NL}}$ defined by Eq. (10)] of the achieved nonlinear AER (red dash-dotted lines) under hybrid control with $\mu_{1}=1, \mu_{2}=1.5, R_{\mathrm{st}}=0.5 Z_{c}$, and $\beta_{\mathrm{NL}}=40$ (a) or $\beta_{\mathrm{NL}}=90$ (c), respectively. The absorption results achieved with both cases of control off (black dashed lines) and of pure linear control (blue dotted lines) are also shown for comparison. For a better demonstration, the pressure amplitudes of the generated second and third harmonics are illustrated in (b) and (d) for the two considered hybrid control cases in parallel with absorption curves, respectively. Sine excitations are performed with a fixed level, to deliver the same incident pressures in front of the AER as presented in Fig. 3 .

and with nonlinear part $i_{\mathrm{NL}}(t)$ obtained by implementing the cubic product of the rear pressure [Eq. (8)]. Figure 7 shows the achieved absorption curve and the corresponding frequency dependence of the generated second and third harmonics, for nonlinear configurations defined by $\beta_{\mathrm{NL}}=40$ [Figs. 7(a) and 7(b)] and $\beta_{\mathrm{NL}}=90$ [Figs. 7(c) and 7(d)], respectively. The control-off case $(i=0)$ and the case of pure linear control $\left(i_{\mathrm{NL}}=0\right)$ are also illustrated in both Figs. 7(a) and 7(c), with black dashed lines and blue dotted lines, respectively.

In comparison with the pure nonlinear control presented in Sec. IV, hybrid control allows for further improvement of the absorption performance. With the presented linear part of the control, the nonlinear parameter can be increased and can even exceed $\beta_{\mathrm{NL}}=90$ without saturation, thus enabling a bandwidth of efficient absorption $(\alpha>0.8$, as explained in Ref. [15]) of around $80 \mathrm{~Hz}$, while increasing the absorption magnitude, with a maximum value up to 0.98 . Moreover, when compared to the pure linear control case, which allows an efficient absorption bandwidth of $40 \mathrm{~Hz}$ and a maximum absorption value of around 0.89 (blue dotted lines in Fig. 7), the

nonlinear part of the proposed hybrid control with the nonlinear parameter set as $\beta_{\mathrm{NL}}=90$ is capable of significantly improving the absorption performance, i.e., doubling the efficient absorption bandwidth and yielding nearly perfect absorption near the target (nonlinear) resonance frequency.

Nevertheless, regarding the third harmonic, although it can be amplified by increasing the value of the nonlinear parameter, the present hybrid control limits the third harmonic generation to an amplitude less than a quarter of that presented with the pure nonlinear control, as is evident when comparing Figs. 7(d) and 7(b) with Fig. 6(b). Under such hybrid control, two maxima are visible in the frequency response of the third harmonic $3 \omega$. The most important one, at a frequency of around $248 \mathrm{~Hz}$, corresponds to the targeted resonance that is prescribed by the linear control law and is shifted slightly due to the nonlinear effect, while the other one appears in the vicinity of the natural resonance and is linked to the mismatch in the mechanical-parameter estimation used for the resonance adjustment through the linear control laws.

Since the ER is never perfectly linear, a second harmonic component is also present, even in the passive case. When the hybrid control is applied, in addition to the two maxima occurring at the same excitation frequencies as the third harmonic, owing to the presence of the resonance, a third one can also be noted at frequencies ranging from half of the natural resonance up to half of the target resonance of the AER. The triggered nonlinear effect favors the second harmonic generation around this range, because of the correspondence between the generated second harmonic and the ER resonances. Conversely, the third harmonic cannot be triggered around $100 \mathrm{~Hz}$, since the applied nonlinear control law cannot play an important role when far from the resonances. Still, when close to the aforementioned two resonance frequencies, the third harmonic prevails over the second harmonic due to the cubic nonlinearity introduced through active control.

Additionally, the influence of the excitation level is also studied. Table II shows the required value of the nonlinear parameter $\beta_{\mathrm{NL}}$ that leads to the same absorption curve as that of Fig. 7(c), as a function of the incident pressure level. One can note that, as the input intensity is decreased, the absorption performance can still be enhanced by increasing the value of the nonlinear parameter. The ability of such hybrid control to improve the sound absorption at low intensities is herein confirmed, regardless of the excitation levels.

TABLE II. The required value of the nonlinear parameter $\beta_{\mathrm{NL}}$ that leads to the same nonlinear absorption curve as that of Fig. 7(c) under different incident pressure levels in front of the AER, achieved by varying the excitation levels.

\begin{tabular}{lccccc}
\hline \hline Incident pressure level (dB) & 88.8 & 91.3 & 94.8 & 97.5 & 99.6 \\
Required value of $\beta_{\mathrm{NL}}$ & 400 & 180 & 90 & 50 & 30 \\
\hline \hline
\end{tabular}


Following the previous configuration, Fig. 8 presents the absorption curve achieved by modifying the linear part of the control law. Here, the reactive parameters are set in order to preserve the same linear target resonance frequency $f_{\text {st }}$ as in Fig. $7\left(\mu_{1}=1\right.$ and $\left.\mu_{2}=1.5\right)$, while varying the target resistance to $R_{\mathrm{st}}=0.3 Z_{c}$ [Figs. 8(a) and 8(b)] and to $R_{\mathrm{st}}=Z_{c}$ [Figs. 8(c) and 8(d)], respectively. The nonlinear parameter is set as high as possible below the saturation threshold. According to the comparison between the three control cases presented in Figs. 7 and 8 , the nonlinear effect is more pronounced as the target resistance $R_{\mathrm{st}}$ decreases. The amplitude of the third harmonic generated by the control with $R_{\mathrm{st}}=0.3 Z_{c}$ shows a maximum that is higher than twice that achieved with $R_{\mathrm{st}}=0.5 Z_{c}$ or $R_{\mathrm{st}}=Z_{c}$, while a lower nonlinear parameter $\left(\beta_{\mathrm{NL}}=70\right.$ instead of 90$)$ is provided in a such control case. Moreover, a rather low resistance allows not only a broadening of the absorption bandwidth, but also an increase of the absorption level. Indeed, as can be seen in Fig. 8(a), the linear control with $R_{\mathrm{st}}=0.3 Z_{c}$ allows a maximum

(a)

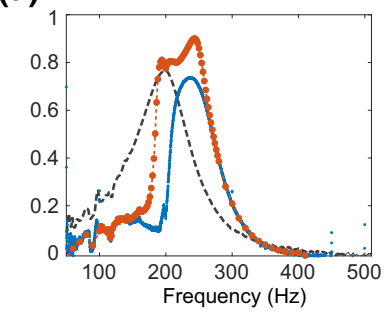

(b)

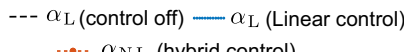
... $\alpha_{\mathrm{NL}}$ (hybrid control)

(c)

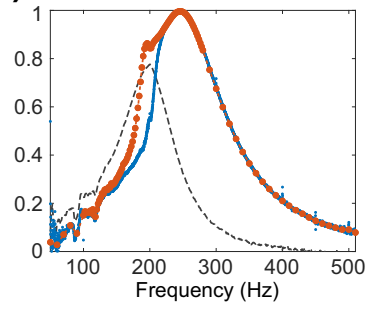

(d)

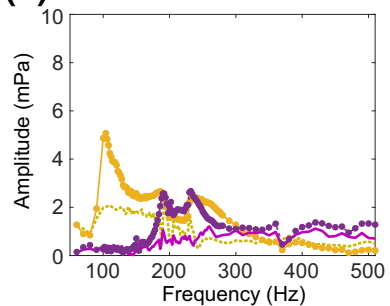

FIG. 8. The absorption curves of the achieved nonlinear AER under different hybrid controls, with the linear design parameters defined as $\mu_{1}=1$ and $\mu_{2}=1.5$ and with the linear target resistance being $R_{\mathrm{st}}=0.3 Z_{c}$ (a) and $R_{\mathrm{st}}=Z_{c}$ (c), respectively. The nonlinear part of the control is applied with an achievable value of the nonlinear parameter set as $\beta_{\mathrm{NL}}=70$ and $\beta_{\mathrm{NL}}=90$, respectively. For both control configurations, higher-harmonic generation is taken into account in the definition of the absorption coefficient $\alpha_{\mathrm{Nl}}$, as described by Eq. (10) and the pressure amplitudes of the second and third harmonics are illustrated in (b) and (d) in parallel with the corresponding absorption curves (a) and (c) for the two control cases, respectively. Pure linear control results with $\beta_{\mathrm{NL}}=0$ (black dashed line) and the control-off case (blue dotted lines) are also presented for comparison with the hybrid control result. magnitude of the absorption coefficient of around 0.73, whereas it can exceed 0.9 with hybrid control, enabling an effective absorption bandwidth $(\alpha>0.8)$ of around $62 \mathrm{~Hz}$.

However, when the target resistance is equal to the specific acoustic impedance of air $\left(R_{\mathrm{st}}=Z_{c}\right)$, although the cubic nonlinearity is triggered to a lesser extent (identified by weak generation of the third harmonic), the final absorption result appears to be optimal. With such a linear control law that provides a nearly total absorption at the targeted resonance frequency, the nonlinear part of the corresponding hybrid control leads predominantly to an increase of the absorption bandwidth. The bandwidth over which effective sound absorption $(\alpha>0.8)$ is achieved is thus extended from $75 \mathrm{~Hz}$ through pure linear control to 95 $\mathrm{Hz}$ through hybrid control, as shown in Fig. 8(c).

Similar to the configurations presented in Figs. 7 and 8, Fig. 9 shows the absorption curves obtained with different reactive parameters of the linear control part $\left(\mu_{1}\right.$ and $\mu_{2}$ ), while maintaining the target resistance to $R_{\mathrm{st}}=0.5 Z_{c}$ and the nonlinear control law with parameter $\beta_{\mathrm{NL}}$ as large as possible, provided that the whole system remains stable (without saturation). (a)

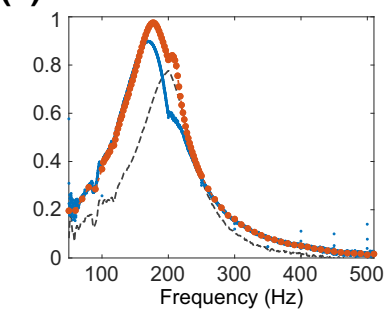

--- $\alpha_{\mathrm{L}}$ (control off) $-\alpha_{\mathrm{L}}$ (linear control) ..--- $\alpha_{\mathrm{NL}}$ (hybrid control)

(c)

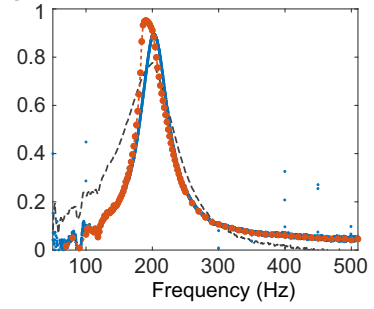

(b)

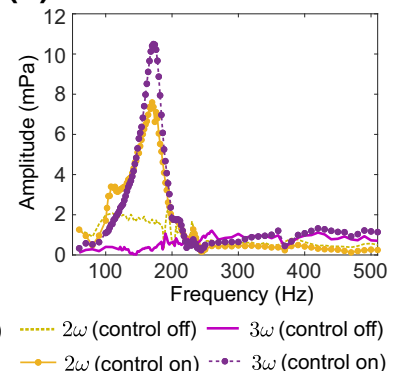

(d)

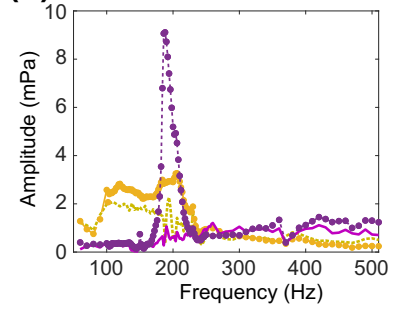

FIG. 9. The absorption curves of the achieved nonlinear AER and the associated pressure amplitudes of the second and third harmonics, by considering a definition of the absorption coefficient suitable for nonlinear systems [Eq. (10)]. Two hybrid control results are presented, identified by the linear parts defined by (a),(b) $\mu_{1}=1, \mu_{2}=0.75$, and $R_{\mathrm{st}}=0.5 Z_{c}$ and (c),(d) $\mu_{1}=$ $\mu_{2}=2$ and $R_{\mathrm{st}}=0.5 Z_{c}$, respectively. The nonlinear part of the control is applied with an achievable value of the nonlinear parameter $\beta_{\mathrm{NL}}$ being (a),(b) $\beta_{\mathrm{NL}}=-30$ and (c),(d) $\beta_{\mathrm{NL}}=30$, respectively. The absorption results of pure linear control with $\beta_{\mathrm{NL}}=0$ (blue dotted lines) and the control-off case (black dashed lines) are also presented for both configurations. 
A linear configuration allowing shifting of the (linear) resonance toward low frequency is first considered and presented in Figs. 9(a) and 9(b), with the linear parameters set as $\mu_{1}=1, \mu_{2}=0.75$, targeting a resonance at around $176 \mathrm{~Hz}$. When the resonance frequency of the AER is linearly tuned below the natural resonance frequency of the passive ER (softening instead of stiffening), the nonlinear parameter $\beta_{\mathrm{NL}}$ needs to be negative to enable improvement of the absorption. With the design parameter $\mu_{2}$ decreasing to 0.5 , an absolute value of 30 for $\beta_{\mathrm{NL}}$ is proved to be consistently achievable in measurement without saturation. Comparing with the cases of $\mu_{1}<\mu_{2}$ [see Figs. 7 and 8], in the present configuration, the nonlinear component of the hybrid control leads to the same trend in absorption improvement, i.e., a bandwidth enlargement and an amplitude increase mainly within the frequency range bounded by the target resonance and the natural resonance of the ER. As a result, even though the current control appears to be less advantageous than the previous controls, a maximum absorption up to 0.98 can still be achieved together with the efficient absorption bandwidth being extended from $36 \mathrm{~Hz}$ through pure linear control to $60 \mathrm{~Hz}$ through hybrid control.

In addition to the previous configuration, Figs. 9(c) and 9(d) present the control results for the linear target impedance defined with $\mu_{1}=\mu_{2}=2$ preserving the (linear) resonance frequency of the passive ER but enabling a higher quality factor (or, in other words, a narrower absorption bandwidth). The target resistance is set to $R_{\mathrm{st}}=0.5 Z_{c}$ as in the previous case of Fig. 9(a). With $\mu_{1}=\mu_{2}>1$, the hybrid control leads to a similar absorption result as the configuration of pure nonlinear control, where $\mu_{1}=\mu_{2}=1$ (see Figs. 5 and 6). The triggered nonlinear effect manifests as a slight enlargement of the absorption bandwidth toward the low-frequency range, enabling the efficient absorption bandwidth to be changed from $194-212 \mathrm{~Hz}$ to $185-206 \mathrm{~Hz}$, and along with an increase of the maximum absorption magnitude from 0.9 to 0.95 . Although it is also possible to broaden the absorption bandwidth with linear control by defining $\mu_{1}=\mu_{2}<1$ $[15,22]$, when such a scheme is implemented in a hybrid control, saturation prevents the nonlinear parameter $\beta_{\mathrm{NL}}$ from being increased to the same level as for the cases $\mu_{1}=\mu_{2} \geq 1$. Thus, in such a configuration, the generated weak nonlinear effect leads to only a tiny improvement of the absorption.

In both configurations presented in Fig. 9, the generated second and third harmonics always present a maximum amplitude at the shifted target-resonance frequency, as in all the previous configurations presented in Figs. 6, 7 and 8. Under the defined cubic nonlinear control law, the third harmonic remains more important than the second one in the frequency range where the nonlinear effect acts on the absorption performance. Conversely, around $100 \mathrm{~Hz}$, the second harmonic is more prominent, since its frequency coincides with either the natural or the target resonance. However, in all considered control configurations, the generated higher harmonics are consistently very weak compared to the fundamental component over the whole frequency range of interest. The maximum pressure amplitude of $0.035 \mathrm{~Pa}$ appears at the third harmonic, corresponding to a proportion of reflected energy only $0.13 \%$.

Hence, according to the results obtained in this section with different hybrid control laws, we conclude that the nonlinear effect enabled via the active control allows for a significant improvement of the absorption performance of the ER, while producing only negligible wave distortion. Depending on the linear part of the control law, the generated nonlinearity can play a role of variable importance, i.e., either in expanding the bandwidth or simultaneously increasing the magnitude and enlarging the bandwidth of the effective absorption. The optimal hybrid control law includes a linear part that slightly shifts the linear maximum absorption and a nonlinear part defined with a nonlinear parameter as high as possible, provided that no saturation occurs. When compared to just the linear or nonlinear active control, the hybrid control presents more advantages in terms of improving the absorption performance of the achieved AER, thus having the potential to be widely used for future low-frequency sound absorption.

\section{CONCLUSION}

Based on an experimental prototype developed for achieving linear active impedance control on a closedbox electrodynamic loudspeaker, in the present work a nonlinear active impedance control is introduced and implemented. Due to the proportionality between the displacement of the loudspeaker diaphragm and the rear pressure, within the low-frequency range of interest $(50-500 \mathrm{~Hz})$, a nonlinear AER with cubic nonlinearity is experimentally achieved, allowing its combination with the already existing linear active ER scheme.

Our study focuses on the absorption performance of the resonator, by first considering a pure nonlinear control and then a hybrid control that combines linear and nonlinear control laws. With a view to fully analyzing the triggered nonlinear effect, an absorption coefficient accounting for the likely generation of higher harmonics is defined. Unlike the other nonlinear mechanisms that require significantly higher pressure levels to enable the nonlinear effect manifestation, such as reported in the literature on NES that is also used for the absorption enhancement, the present control architectures allow for improving sound absorption at much lower excitation levels, while producing negligible distortion. Compared to the employed passive SDOF ER, which presents a maximum absorption coefficient of about 0.77 at its natural resonance frequency, under an incident sound pressure level of around $94.8 \mathrm{~dB}$ (around 1.1 Pa) in front of the resonator, a considerable 
increase in the absorption coefficient, to above 0.8 , can be achieved through the proposed hybrid control within a frequency range larger than $80 \mathrm{~Hz}$, along with only $0.13 \%$ of the energy being reflected through higher harmonics.

In the present work, a cubic nonlinear control law on the diaphragm displacement is taken into account. In order to ensure that the performed control operates as defined, a time-domain integration method is used to simulate the full problem. Relatively good agreement is found between the experimental results and the simulation implementations. Such a nonlinear control law is also presented as an active way of achieving a cubic nonlinear stiffness on the resonator. Additionally, the proposed nonlinear active control not only facilitates the generation of nonlinearities on the ER but also allows them to be adjustable and reprogrammable, which is very difficult to obtain using mechanical nonlinearities.

Nevertheless, since the reported nonlinear and hybrid control results strongly depend on the passive acoustical parameters of the considered ER, i.e., the mass $M_{\text {as }}$, the compliance $C_{\mathrm{ac}}$, the resistance $R_{\mathrm{as}}$, and the force factor $B l$, which are numerically extracted from two impedance measurements with different electric loads, the performance could be further improved by additional measurements; for instance, by evaluating the effective area of the diaphragm $S_{d}$. Alternatively, the hybrid control law is investigated herein, i.e., with a linear part that restricts the ER such that it has a single degree of freedom and with a nonlinear part focusing on the cubic displacement nonlinearity. In the future, other types of nonlinearity could be achieved through the proposed experimental prototype and could be combined with active linear multiple-degrees-offreedom ER, with the aim of further improving the sound absorption.

As a perspective, such an active control scheme could be employed in the design of acoustic metamaterials, with a view to achieving nontrivial wave phenomena. Indeed, a unit cell implementing the reported active control scheme, with two microphones (one sensing the front pressure and another the rear pressure related to the diaphragm displacement), could, for instance, intrinsically present a negative effective bulk modulus. By combining a nonlinear law with such a linear active control, a new family of nonlinear active metamaterials with potentially larger bandwidth or multistable functionalities could be developed.

\section{ACKNOWLEDGMENTS}

We wish to thank Dr. Sami Karkar for his precious advice on the implementation of nonlinear stiffness.

[1] M. A. Biot, Theory of propagation of elastic waves in a fluid-saturated porous solid. I. Low-frequency range, J. Acoust. Soc. Am. 28, 168 (1956).
[2] J. Allard and N. Atalla, Propagation of Sound in Porous Media: Modeling Sound Absorbing Materials (John Wiley \& Sons, 2009), 2nd ed.

[3] L. D. Landau and E. M. Lifshiz, in Electrodynamics of Continuous Media, Course of Theoretical Physics, Vol. 8 (Pergamon, Amsterdam, Chichester, 1984), 2nd ed., p. 257.

[4] R. M. Fano, Theoretical limitations on the broadband matching of arbitrary impedances, J. Franklin Inst. 249, 57 (1950).

[5] K. N. Rozanov, Ultimate thickness to bandwidth ratio of radar absorbers, IEEE Trans. Antennas Propag. 48, 1230 (2000).

[6] C. Sohl, M. Gustafsson, and G. Kristensson, The integrated extinction for broadband scattering of acoustic waves, J. Acoust. Soc. Am. 6, 3206 (2007).

[7] M. Yang, S. Chen, C. Fu, and P. Sheng, Optimal soundabsorbing structures, Mater. Horiz. 4, 673 (2017).

[8] H. W. Bode, Network Analysis and Feedback Amplifier Design (Van Nostrand, New York, 1945).

[9] D. M. Pozar, Microwave Engineering (John Wiley \& Sons, New York, 1998), 2nd ed., p. 295.

[10] O. Acher, J. M. L. Bernard, P. Maréchal, A. Bardaine, and F. Levassort, Fundamental constraints on the performance of broadband ultrasonic matching structures and absorbers, J. Acoust. Soc. Am. 125, 1995 (2009).

[11] F. Monticone and A. Alù, Invisibility exposed: Physical bounds on passive cloaking, Optica 3, 718 (2016).

[12] P. Chen, C. Argyropoulos, and A. Alù, Broadening the Cloaking Bandwidth with Non-Foster Metasurfaces, Phys. Rev. Lett. 111, 233001 (2013).

[13] S. E. Sussman-Fort and R. M. Rudish, Non-impedance matching of electrically-small antennas, IEEE Trans. Antennas Propag. 57, 2230 (2009).

[14] A. Shlivinski and Y. Hadad, Beyond the Bode-Fano Bound: Wideband Impedance Matching for Short Pulses Using Temporal Switching of Transmission-Line Parameters, Phys. Rev. Lett. 121, 204301 (2018).

[15] E. Rivet, S. Karkar, and H. Lissek, Broadband lowfrequency electroacoustic absorbers through hybrid sensor/shunt-based impedance control, IEEE Trans. Control Syst. Technol. 25, 63 (2017).

[16] H. Lissek, R. Boulandet, and R. Fleury, Electroacoustic absorbers: Bridging the gap between shunt loudspeakers and active sound absorption, J. Acoust. Soc. Am. 129, 2968 (2011).

[17] E. Rivet, S. Karkar, and H. Lissek, Multi-degree-offreedom low-frequency electroacoustic absorbers through coupled resonators, Appl. Acoust. 132, 109 (2018).

[18] E. Rivet, S. Karkar, and H. Lissek, On the optimisation of multi-degree-of-freedom acoustic impedances of low-frequency electroacoustic absorbers for room modal equalisation, Acta Acust. United Ac. 103, 1025 (2017).

[19] R. Boulandet and H. Lissek, Optimization of electroacoustic absorbers by means of designed experiments, Appl. Acoust. 71, 830 (2010).

[20] T. T. Koutserimpas, E. Rivet, H. Lissek, and R. Fleury, Active Acoustic Resonators with Reconfigurable Resonance Frequency, Absorption, and Bandwidth, Phys. Rev. Appl. 12, 054064 (2019). 
[21] E. Rivet, S. Karkar, H. Lissek, T. N. Thorsen, and V. Adam, in 140th Audio Engineering Society Convention, Paris, France (2016).

[22] E. Rivet, Ph.D. thesis, Ecole Polytechnique Fédérale de Lausanne, 2016.

[23] R. Boulandet, H. Lissek, S. Karkar, M. Collet, G. Matten, M. Ouisse, and M. Versaevel, Duct modes damping through an adjustable electroacoustic liner under grazing incidence, J. Sound Vib. 426, 19 (2018).

[24] F. Zangeneh-Nejad and R. Fleury, Active times for acoustic metamaterials, Rev. Phys. 4, 100031 (2019).

[25] B.-I. Popa, D. Shinde, A. Konneker, and S. A. Cummer, Active acoustic metamaterials reconfigurable in real time, Phys. Rev. B 91, 220303 (2015).

[26] A. Bergamini, T. Delpero, L. D. Simoni, L. D. Lillo, M. Ruzzene, and P. Ermanni, Phononic crystal with adaptive connectivity, Adv. Mater. 26, 1343 (2014).

[27] S. A. Cummer, J. Christensen, and A. Alù, Controlling sound with acoustic metamaterials, Nat. Rev. Mater. 1, 16001 (2016).

[28] R. Fleury, D. Sounas, and A. Alù, An invisible acoustic sensor based on parity-time symmetry, Nat. Commun. 6, 5905 (2015).

[29] Y. Aurégan and V. Pagneux, $\mathcal{P} \mathcal{T}$-Symmetric Scattering in Flow Duct Acoustics, Phys. Rev. Lett. 118, 174301 (2017).

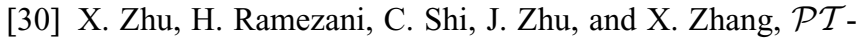
Symmetric Acoustics, Phys. Rev. X 4, 031042 (2014).

[31] H. Lü, S. K. Özdemir, L.-M. Kuang, F. Nori, and H. Jing, Exceptional Points in Random-Defect Phonon Lasers, Phys. Rev. Appl. 8, 044020 (2017).

[32] G. Ma, X. Fan, P. Sheng, and M. Fink, Shaping reverberating sound fields with an actively tunable metasurface, Proc. Natl. Acad. Sci. U.S.A. 115, 6638 (2018).

[33] M. Dupré, P. del Hougne, M. Fink, F. Lemoult, and G. Lerosey, Wave-Field Shaping in Cavities: Waves Trapped in a Box with Controllable Boundaries, Phys. Rev. Lett. 115, 017701 (2015).

[34] H. Lissek, E. Rivet, T. Laurence, and R. Fleury, Toward wideband steerable acoustic metasurfaces with arrays of active electroacoustic resonators, J. Appl. Phys. 123, 091714 (2018).

[35] E. Rivet, A. Brandstötter, K. G. Makris, H. Lissek, S. Rotter, and R. Fleury, Constant-pressure sound waves in nonHermitian disordered media, Nat. Phys. 14, 942 (2018).

[36] K. G. Makris, Z. H. Musslimani, D. N. Christodoulides, and S. Rotter, Constant-intensity waves and their modulation instability in non-Hermitian potentials, Nat. Commun. 6, 7257 (2015).

[37] M. Furstoss, D. Thenail, and M. A. Galland, Surface impedance control for sound absorption: Direct and hybrid passive/active strategies, J. Sound Vib. 203, 219 (1997).

[38] A. F. Vakakis and O. V. Gendelman, Energy pumping in nonlinear mechanical oscillators: Part II. Resonance capture, J. Appl. Mech. 68, 42 (2000).

[39] O. V. Gendelman, Transition of energy to a nonlinear localized mode in a highly asymmetric system of two oscillators, Nonlinear Dyn. 25, 237 (2001).
[40] Y. Starosvetsky and O. V. Gendelman, Dynamics of a strongly nonlinear vibration absorber coupled to a harmonically excited two-degree-of-freedom system, J. Sound Vib. 312, 234 (2008).

[41] E. Gourdon, N. A. Alexander, C. A. Taylor, C. H. Lamarque, and S. Pernot, Nonlinear energy pumping under transient forcing with strongly nonlinear coupling: Theoretical and experimental results, J. Sound Vib. 300, 522 (2007).

[42] G. Sigalov, O. V. Gendelman, M. A. AL-Shudeifat, L. I. Manevitch, A. F. Vakakis, and L. A. Bergman, Resonance captures and targeted energy transfers in an inertiallycoupled rotational nonlinear energy sink, Nonlinear Dyn. 69, 1693 (2012).

[43] R. Bellet, B. Cochelin, P. Herzog, and P.-O. Mattei, Experimental study of targeted energy transfer from an acoustic system to a nonlinear membrane absorber, J. Sound Vib. 329, 2768 (2010).

[44] R. Mariani, S. Bellizzi, B. Cochelin, P. Herzog, and P.-O. Mattei, Toward an adjustable nonlinear low frequency acoustic absorber, J. Sound Vib. 330, 5245 (2011).

[45] R. Côte, M. Pachebat, and S. Bellizzi, Experimental evidence of simultaneous multi-resonance noise reduction using an absorber with essential nonlinearity under two excitation frequencies, J. Sound Vib. 333, 5057 (2014).

[46] P. Y. Bryk, S. Bellizzi, and R. Côte, Experimental study of a hybrid electro-acoustic nonlinear membrane absorber, J. Sound Vib. 424, 224 (2018).

[47] X. Guo, V. E. Gusev, K. Bertoldi, and V. Tournat, Manipulating acoustic wave reflection by a nonlinear elastic metasurface, J. Appl. Phys. 123, 124901 (2018).

[48] X. Guo, V. E. Gusev, B. Deng, K. Bertoldi, and V. Tournat, Frequency-doubling effect in acoustic reflection by a nonlinear, architected rotating-square metasurface, Phys. Rev. E 99, 052209 (2019).

[49] S. G. Konarski, M. R. Haberman, and M. F. Hamilton, Frequency-dependent behavior of media containing pre-strained nonlinear inclusions: Application to nonlinear acoustic metamaterials, J. Acoust. Soc. Am. 144, 3022 (2018).

[50] F. Zangeneh-Nejad and R. Fleury, Nonlinear SecondOrder Topological Insulators, Phys. Rev. Lett. 123, 053902 (2019).

[51] V. V. Konotopand, J. Yang, and D. A. Zezyulin, Nonlinear waves in $\mathcal{P} \mathcal{T}$-symmetric systems, Rev. Mod. Phys. 88, 035002 (2016).

[52] B.-I. Popa and S. A. Cummer, Non-reciprocal and highly nonlinear active acoustic metamaterials, Nat. Commun. 5, 3398 (2014).

[53] D. Bitar, E. Gourdon, C.-H. Lamarque, and M. Collet, Shunt loudspeaker using nonlinear energy sink, J. Sound Vib. 456, 254 (2019).

[54] E. Hairer, S. P. Norsett, and G. Wanner, in Solving Ordinary Differential Equations I: Nonstiff Problems (Springer, Berlin, Heidelberg, 1993), p. 129. 